\title{
COHOMOLOGICAL DIMENSION AND METRIZABLE SPACES. II
}

\author{
JERZY DYDAK
}

\begin{abstract}
The main result of the first part of the paper is a generalization of the classical result of Menger-Urysohn : $\operatorname{dim}(A \cup B) \leq \operatorname{dim} A+\operatorname{dim} B+1$.

Theorem. Suppose $A, B$ are subsets of a metrizable space and $K$ and $L$ are $C W$ complexes. If $K$ is an absolute extensor for $A$ and $L$ is an absolute extensor for $B$, then the join $K * L$ is an absolute extensor for $A \cup B$.
\end{abstract}

As an application we prove the following analogue of the Menger-Urysohn Theorem for cohomological dimension:

Theorem. Suppose A, B are subsets of a metrizable space. Then

$$
\operatorname{dim}_{\mathbf{R}}(A \cup B) \leq \operatorname{dim}_{\mathbf{R}} A+\operatorname{dim}_{\mathbf{R}} B+1
$$

for any ring $\mathbf{R}$ with unity and

$$
\operatorname{dim}_{G}(A \cup B) \leq \operatorname{dim}_{G} A+\operatorname{dim}_{G} B+2
$$

for any abelian group $G$.

The second part of the paper is devoted to the question of existence of universal spaces:

Theorem. Suppose $\left\{K_{i}\right\}_{i \geq 1}$ is a sequence of $C W$ complexes homotopy dominated by finite $C W$ complexes. Then

a. Given a separable, metrizable space $Y$ such that $K_{i} \in A E(Y), i \geq 1$, there exists a metrizable compactification $c(Y)$ of $Y$ such that $K_{i} \in$ $A E(c(Y)), i \geq 1$.

b. There is a universal space of the class of all compact metrizable spaces $Y$ such that $K_{i} \in A E(Y)$ for all $i \geq 1$.

c. There is a completely metrizable and separable space $Z$ such that $K_{i} \in$ $A E(Z)$ for all $i \geq 1$ with the property that any completely metrizable and separable space $Z^{\prime}$ with $K_{i} \in A E\left(Z^{\prime}\right)$ for all $i \geq 1$ embeds in $Z$ as a closed subset.

\section{INTRODUCTION}

The paper is devoted to addressing the following questions in cohomological dimension theory:

Received by the editors December 11, 1992 and, in revised form, May 3, 1995

1991 Mathematics Subject Classification. Primary 55M11, 54F45.

Key words and phrases. Dimension, cohomological dimension, Menger-Urysohn Theorem, absolute extensors, Eilenberg-Mac Lane spaces, universal spaces, compactifications.

Supported in part by a grant from the National Science Foundation 
Problem 1 (see $[\mathrm{Ku}])$. Does

$$
\operatorname{dim}_{G}(A \cup B) \leq \operatorname{dim}_{G} A+\operatorname{dim}_{G} B+1
$$

hold for any group $G$ and any subsets $A$ and $B$ of a metrizable space?

Problem 2 (see [We] for $G=\mathbf{Z}$ ). Suppose $n \geq 1$ and $G$ is an abelian group. Is there a universal compactum in the class of compacta $\left\{X \mid \operatorname{dim}_{G} X \leq n\right\}$ ?

Problem 3. Suppose $n \geq 1, G$ is an abelian group and $X$ is a metrizable and separable space such that $\operatorname{dim}_{G} X \leq n$. Is there a completion $X^{\prime}$ of $X$ such that $\operatorname{dim}_{G} X^{\prime} \leq n$ ?

Problems 1-3 are part of a general plan to construct cohomological dimension theory, formally parallel to the theory of covering dimension. They were motivated by analogous results involving covering dimension.

Why not be more ambitious and attempt to create a theory encompassing both covering dimension and cohomological dimension? Such an idea was set forth by A. Dranishnikov at a recent workshop on cohomological dimension theory. He coined the term "Extension Theory" for the new theory.

With this idea in mind we prove the following result:

Theorem A. Suppose $A, B$ are subsets of a metrizable space and $K$ and $L$ are $C W$ complexes. If $K$ is an absolute extensor for $A$ and $L$ is an absolute extensor for $B$, then the join $K * L$ is an absolute extensor for $A \cup B$.

Obviously, Theorem A generalizes the classical Urysohn-Menger formula:

$$
\operatorname{dim}(A \cup B) \leq \operatorname{dim} A+\operatorname{dim} B+1
$$

as $\operatorname{dim} X \leq n$ means $S^{n} \in A E(X)$ and $S^{n} * S^{m}=S^{n+m+1}$. At the same time, using Theorem A, one can derive a complete solution to Problem 1:

Theorem B. Suppose A,B are subsets of a metrizable space. Then

$$
\operatorname{dim}_{\mathbf{R}}(A \cup B) \leq \operatorname{dim}_{\mathbf{R}} A+\operatorname{dim}_{\mathbf{R}} B+1
$$

for any ring $\mathbf{R}$ with unity, and

$$
\operatorname{dim}_{G}(A \cup B) \leq \operatorname{dim}_{G} A+\operatorname{dim}_{G} B+2
$$

for any abelian group $G$.

Special cases of Theorem B have been established by L.Rubin $[R u](\mathbf{R}=\mathbf{Z})$, Dydak-Walsh $\left[\mathrm{D}-\mathrm{W}_{1}\right](\mathbf{R}=\mathbf{Z} / \mathbf{p}$ or $\mathbf{R}$ is a subring of the ring of rationals $\mathbf{Q}$, and $\left.\operatorname{dim}_{\mathbf{R}} A, \operatorname{dim}_{\mathbf{R}} B \geq 2\right)$ and Dranishnikov-Repovš $[\mathrm{D}-\mathrm{R}](\mathbf{R}=\mathbf{Z} / \mathbf{p}$ or $\mathbf{R}$ is a subring of $\mathbf{Q}, \operatorname{dim}(A \cup B)$ is finite and $\operatorname{dim}_{\mathbf{R}} A=1$ or $\left.\operatorname{dim}_{\mathbf{R}} B=1\right)$. Notice that the estimates of Theorem B cannot be improved. Indeed, Dranishnikov, Repovš and Ščepin [D-RS] found an example such that $G=\mathbf{Q} / \mathbf{Z}$ and $\operatorname{dim}_{G}(A \cup B)=\operatorname{dim}_{G} A+\operatorname{dim}_{G} B+2$.

Here are problems which belong to the theory unifying both the covering dimension and cohomological dimension:

Problem 4. Characterize $C W$ complexes $K$ such that for any separable metric space $X$ with $K \in A E(X)$, there is a completion $X^{\prime}$ of $X$ with $K \in A E\left(X^{\prime}\right)$. 
Problem 5. Characterize $C W$ complexes $K$ such that for any separable metric space $X$ with $K \in A E(X)$, there is a compactification $X^{\prime}$ of $X$ with $K \in A E\left(X^{\prime}\right)$.

Problem 6. Characterize $C W$ complexes $K$ such that the class

$\{X$ is a separable metric space with $K \in A E(X)\}$

has a universal space.

Problem 7. Characterize $C W$ complexes $K$ such that the class

$\{X$ is a compact metrizable space with $K \in A E(X)\}$

has a universal space.

In the second part of the paper we prove a theorem which implies all the results known up to now pertaining to Problems 4-7. This is done using a technique from $\left[\mathrm{Dy}_{4}\right]$.

Problem 8. Suppose $X$ is a compactum (separable and metrizable space) and $K, L$ are $C W$ complexes such that $K * L \in A E(X)$. Is there a subset $A$ of $X$ such that $K \in A E(A)$ and $L \in A E(X-A)$ ?

A positive answer to Problem 8 would generalize the well-known result that $n$-dimensional spaces can be expressed as a union of $n+1$ of its 0 -dimensional subsets.

Finally, we propose the following problem as a generalization of the well-known inequality $\operatorname{dim}(X \times Y) \leq \operatorname{dim} X+\operatorname{dim} Y$ :

Problem 9. Suppose $X, Y$ are separable metric (compact) and $K \in A E(X)$, $L \in A E(Y)$ are $C W$ complexes. Is $K \wedge L \in A E(X \times Y)$ ?

A positive solution to Problem 9 in the compact case would recover all that is known about cohomological dimension of cartesian products (see $[\mathrm{Ku}]$ ). In case of arbitrary $X$ and $Y$, it would extend the known results from the compact case. It is interesting that so far only $\operatorname{dim}_{\mathbf{Z}}(X \times Y) \leq \operatorname{dim}_{\mathbf{Z}} X+\operatorname{dim}_{\mathbf{Z}} Y$ was established by L. Rubin using cell-like maps [R-S].

The author is grateful to Sasha Dranishnikov and Wojciech Olszewski for pointing out errors in the first draft of the paper. Also, the author would like to acknowledge the help of the referee, whose numerous comments resulted in improvement of the paper.

\section{Cohomological dimension of unions of Sets}

Recall that $Y$ is called an absolute extensor (see [Hu]) of $X$ (notation: $Y \in$ $A E(X))$ provided any map $f: A \rightarrow Y, A \subset X$ closed, extends over $X$. The following Proposition is proved in [Wa, p.107] in the case of $K$ being a $K(\mathbf{Z}, n)$ and $X$ being metrizable separable and in [Dr 2 , Proposition 2] for $Y$ being open in $X$ :

Proposition 1.1. Suppose $X$ is a metrizable space. If $K \in A E(X)$ is a $C W$ complex, then $K \in A E(Y)$ for any subset $Y$ of $X$.

Proof. Suppose $A$ is a closed subset of $Y$ and $f: A \rightarrow K$. Extend $f$, up to homotopy, over an open neighborhood $U$ of $A$ in $X$ (see [M-S], Corollary 3 and 
Remark 3 on p.81). Let $F: U \rightarrow K$ be a homotopy extension of $f$. Let $V=$ $X-\operatorname{cl}_{X}(A) \cap(X-U)$. Notice that $Y \subset V\left(\right.$ as $\left.Y \cap c_{X}(A) \cap(X-U)=A \cap(X-U)=\emptyset\right)$ and $\operatorname{cl}_{V}(A) \subset U\left(\right.$ as $\left.\operatorname{cl}_{V}(A) \cap(X-U) \subset \operatorname{cl}_{X}(A) \cap(X-U) \subset X-V\right)$. Since $K \in A E(V)$ by $\left[\operatorname{Dr}_{2}\right.$, Proposition 2], $F \mid \operatorname{cl}_{V}(A)$ can be extended over $V$. That extension, when restricted to $Y$, gives a homotopy extension of $f$.

Definition. Given two simplicial complexes $K$ and $L$, their simplicial join $K * L$ is formed by declaring $\left.<s_{0}, \ldots, s_{k}, t_{0}, \ldots, t_{l}\right\rangle$ to be its simplex iff $\left\langle s_{0}, \ldots, s_{k}\right\rangle$ is a simplex in $K$ and $\left\langle t_{0}, \ldots, t_{l}\right\rangle$ is a simplex in $L$. It carries a natural $\mathrm{CW}$ structure; its cells are of the form $s * \sigma$, where $s$ is a simplex of $K$ and $\sigma$ is a simplex of $L$. Notice that $K * L$ with the $\mathrm{CW}$ topology is homeomorphic to the union $M\left(\pi_{K}\right) \cup M\left(\pi_{L}\right)$ of mapping cylinders of projections $\pi_{K}: K \times L \rightarrow K$ and $\pi_{L}: K \times L \rightarrow L$, where we assume $M\left(\pi_{K}\right) \cap M\left(\pi_{L}\right)=K \times L$.

Theorem 1.2. Suppose $A, B$ are subsets of a metrizable space and $K$ and $L$ are simplicial complexes. If $K$ is an absolute extensor for $A$ and $L$ is an absolute extensor for $B$, then the join $K * L$ is an absolute extensor for $A \cup B$.

Proof. Since the identity $M \rightarrow M_{m}$ from a simplicial complex equipped with the CW topology to itself equipped with the metric topology is a homotopy equivalence (see [M-S, Theorem 10 on p.302], we will consider all simplicial complexes to be equipped with the metric topology. Suppose $C$ is a closed subset of $X=A \cup B$ and $f: C \rightarrow K * L$. Notice that $f$ defines two closed, disjoint subsets $C_{K}=f^{-1}(K)$, $C_{L}=f^{-1}(L)$ of $C$ and maps $f_{K}: C-C_{L} \rightarrow K, f_{L}: C-C_{K} \rightarrow L, \alpha: C \rightarrow[0,1]$ such that:

1. $\alpha^{-1}(0)=C_{K}, \alpha^{-1}(1)=C_{L}$,

2. $f(x)=(1-\alpha(x)) \cdot f_{K}(x)+\alpha(x) \cdot f_{L}(x)$ for all $x \in C$.

Indeed, each point $x$ of a simplicial complex $M$ can be uniquely written as $x=\sum_{v \in M^{(0)}} \phi_{v}(x) \cdot v$, where $M^{(0)}$ is the set of vertices of $M\left(\left\{\phi_{v}(x)\right\}\right.$ are called barycentric coordinates of $x$ ). We define $\alpha(x)$ as $\sum_{v \in L^{(0)}} \phi_{v}(f(x)) ; f_{K}(x)$ is defined as $\left(\sum_{v \in K^{(0)}} \phi_{v}(f(x)) \cdot v\right) /(1-\alpha(x))$ and $f_{L}(x)$ as $\left(\sum_{v \in L^{(0)}} \phi_{v}(f(x)) \cdot v\right) /(\alpha(x))$.

Since $K \in A E\left(A-C_{L}\right)$ by Proposition 1.1, $f_{K}$ extends over $C \cup A-C_{L}$. Consider a homotopy extension $g_{K}: U_{A} \rightarrow K$ of $f_{K}$ over a neighborhood $U_{A}$ of $C \cup A-C_{L}$ in $X-C_{L}$ (see [M-S], Corollary 3 and Remark 3 on p.81). Since $C-C_{L}$ is closed in $U_{A}$, we may assume that $g_{K}$ is an actual extension of $f_{K}: C-C_{L} \rightarrow K$. Similarly, let $g_{L}: U_{B} \rightarrow L$ be an extension of $f_{L}$ over a neighborhood $U_{B}$ of $C \cup B-C_{K}$ in $X-C_{K}$. Notice that $X=U_{A} \cup U_{B}$. Let $\beta: X \rightarrow[0,1]$ be an extension of $\alpha$ such that $\beta^{-1}(0)=X-U_{B}$ and $\beta^{-1}(1)=X-U_{A}$. Define $f^{\prime}: X \rightarrow K * L$ by

$$
\begin{gathered}
f^{\prime}(x)=(1-\beta(x)) \cdot g_{K}(x)+\beta(x) \cdot g_{L}(x) \text { for all } x \in U_{A} \cap U_{B}, \\
f^{\prime}(x)=g_{K}(x) \text { for all } x \in U_{A}-U_{B}
\end{gathered}
$$

and

$$
f^{\prime}(x)=g_{L}(x) \text { for all } x \in U_{B}-U_{A} .
$$

Notice that $f^{\prime}$ is an extension of $f$. To prove the continuity of $f^{\prime}$ we need to show that $\phi_{v} f^{\prime}$ is continuous for all vertices $v$ of $K * L$ (see [M-S, Theorem 8 on p. 301]). Without loss of generality we may assume that $v \in K^{(0)}$. Then,

$$
\phi_{v} f^{\prime}(x)=(1-\beta(x)) \cdot \phi_{v} g_{K}(x) \text { for all } x \in U_{A}
$$


and

$$
\phi_{v} f^{\prime}(x)=0 \text { for all } x \in U_{B}-U_{A} .
$$

Clearly, $\phi_{v} f^{\prime} \mid U_{A}$ is continuous. If $x_{0} \in U_{B}-U_{A}$ and $x_{n} \rightarrow x_{0}, x_{n} \in U_{A}$, then $\beta\left(x_{n}\right) \rightarrow 1$ and $0 \leq \phi_{v} g_{K}\left(x_{n}\right) \leq 1$. Consequently, $\phi_{v} f^{\prime}\left(x_{n}\right) \rightarrow 0=\phi_{v} f^{\prime}\left(x_{0}\right)$.

Remark. Since $\operatorname{dim} X \leq n$ means that $S^{n} \in A E(X)$ for any metrizable space $X$ (see [Wa]) and $S^{n} * S^{m}=S^{n+m+1}$, Theorem 1.2 is a generalization of the MengerUrysohn Theorem.

Theorem 1.3. Suppose $A, B$ are subsets of a metrizable space and $G, H$ are abelian groups. Then

$$
\operatorname{dim}_{G \otimes H}(A \cup B) \leq \operatorname{dim}_{G} A+\operatorname{dim}_{H} B+1
$$

and

$$
\operatorname{dim}_{G * H}(A \cup B) \leq \operatorname{dim}_{G} A+\operatorname{dim}_{H} B+2 .
$$

Proof. Suppose $G, H \neq 0$ are abelian groups and $\operatorname{dim}_{G} A=m \geq \operatorname{dim}_{H} B=n$. If $m=n=0$, then $\operatorname{dim} A=\operatorname{dim} B=0$ and Theorem 1.3 reduces to the UrysohnMenger Theorem. So assume $m>0$. Thus, $K \in A E(A)$ for any $K$ being a $K(G, m)$ and $L \in A E(B)$ for any $L$ being a $K(H, n)\left(L=S^{0}\right.$ if $\left.n=0\right)$. By Theorem 1.2, $K * L \in A E(A \cup B)$. Choose base points $k \in K$ and $l \in L$. Since $K * L$ can be expressed as the union $M\left(\pi_{K}\right) \cup M\left(\pi_{L}\right)$ of mapping cylinders of projections $\pi_{K}: K \times L \rightarrow K$ and $\pi_{L}: K \times L \rightarrow L$ (we assume $M\left(\pi_{K}\right) \cap M\left(\pi_{L}\right)=K \times L$ ), it contains a contractible subcomplex

$$
M\left(\pi_{K} \mid K \vee L\right) \cup M\left(\pi_{L} \mid K \vee L\right),
$$

where $K \vee L=K \times l \cup k \times L$, such that

$$
K * L /\left(M\left(\pi_{K} \mid K \vee L\right) \cup M\left(\pi_{L} \mid K \vee L\right)\right) \approx \Sigma(K \wedge L)
$$

Thus, $K * L$ is homotopy equivalent to the suspension $\Sigma(K \wedge L)$ of the smash product $K \wedge L=K \times L / K \vee L$ of $K$ and $L$.

Assume $n>0$. By the Künneth Formula ([Sp], p.235) there is an exact sequence

$$
\begin{aligned}
0 & \rightarrow[H(K, k ; \mathbf{Z}) \otimes H(L, l ; \mathbf{Z})]_{q} \rightarrow H_{q}((K, k) \times(L, l) ; \mathbf{Z}) \\
& \rightarrow[H(K, k ; \mathbf{Z}) * H(L, l ; \mathbf{Z})]_{q-1} \rightarrow 0
\end{aligned}
$$

for all $q$. If $q=m+n$, then

$$
[H(K, k ; \mathbf{Z}) * H(L, l ; \mathbf{Z})]_{q-1}=0
$$

and

$$
[H(K, k ; \mathbf{Z}) \otimes H(L, l ; \mathbf{Z})]_{q}=H_{m}(K, k ; \mathbf{Z}) \otimes H_{n}(L, l ; \mathbf{Z})=G \otimes H .
$$

Consequently,

$H_{m+n+1}(K * L, \mathbf{Z})=H_{m+n}(K \times L, K \vee L ; \mathbf{Z})=H_{m+n}((K, k) \times(L, l) ; \mathbf{Z})=G \otimes H$. 
If $q=m+n+1$, then $[H(K, k ; \mathbf{Z}) * H(L, l ; \mathbf{Z})]_{q-1}=G * H$. Consequently,

$H_{m+n+2}(K * L, \mathbf{Z})=H_{m+n+1}(K \times L, K \vee L ; \mathbf{Z})=H_{m+n+1}((K, k) \times(L, l) ; \mathbf{Z})$

contains $G * H$ is a direct summand. Now, by Theorem 3.4,

and

$$
\operatorname{dim}_{G \otimes H}(A \cup B) \leq m+n+1
$$

$$
\operatorname{dim}_{G * H}(A \cup B) \leq m+n+2 .
$$

If $n=0$, then $H_{m+1}(K * L, \mathbf{Z})=G$, so (by Theorem 3.4), $\operatorname{dim}_{G}(A \cup B) \leq m+1$. Since $\operatorname{dim}_{G \otimes H}(A \cup B) \leq \operatorname{dim}_{G}(A \cup B)\left(\right.$ see $\left.\left[\mathrm{Dy}_{1}\right]\right), \operatorname{dim}_{G \otimes H}(A \cup B) \leq m+1$.

It remains to prove that $\operatorname{dim}_{G * H}(A \cup B) \leq m+2$. This follows from the fact that $\mathbf{p}$-Tor $(G * H) \neq 0$ implies $\mathbf{p}$-Tor $G \neq 0$ and from the Bockstein Inequality $\operatorname{dim}_{\mathbf{Z} / \mathbf{p}} X \leq \operatorname{dim}_{\mathbf{Z} / \mathbf{p}^{\infty}} X+1$ (see $\left.[\mathrm{Ku}]\right)$ for any paracompact space $X$.

Theorem 1.4. Suppose $A, B$ are subsets of a metrizable space. Then

$$
\operatorname{dim}_{\mathbf{R}}(A \cup B) \leq \operatorname{dim}_{\mathbf{R}} A+\operatorname{dim}_{\mathbf{R}} B+1
$$

for any ring $\mathbf{R}$ with unity, and

$$
\operatorname{dim}_{G}(A \cup B) \leq \operatorname{dim}_{G} A+\operatorname{dim}_{G} B+2
$$

for any abelian group $G$.

Proof. If $\mathbf{R}$ is a ring with unity 1 , then $\mathbf{R}$ is a retract of $\mathbf{R} \otimes \mathbf{z} \mathbf{R}$. Indeed, the homomorphism $m: \mathbf{R} \otimes \mathbf{z} \mathbf{R} \rightarrow \mathbf{R}$ induced by the multiplication $\mathbf{R} \times \mathbf{R} \rightarrow \mathbf{R}$ is a left inverse of $i d \otimes 1: \mathbf{R} \rightarrow \mathbf{R} \otimes_{\mathbf{Z}} \mathbf{R}$. Thus,

$$
\operatorname{dim}_{\mathbf{R}}(A \cup B) \leq \operatorname{dim}_{\mathbf{R}} A+\operatorname{dim}_{\mathbf{R}} B+1 .
$$

Let $G$ be an abelian group. Since

$$
\operatorname{dim}_{G} X=\max \left(\operatorname{dim}_{G / \text { Tor } G} X, \operatorname{dim}_{\text {Tor } G} X\right)
$$

(see $\left.\left[\mathrm{Dy}_{1}\right]\right)$, it suffices to prove

$$
\operatorname{dim}_{G}(A \cup B) \leq \operatorname{dim}_{G} A+\operatorname{dim}_{G} B+2
$$

first for torsion groups and then for torsion-free groups.

Since $\mathbf{Z} / \mathbf{p}$ is a ring with unity, the case $G=\mathbf{Z} / \mathbf{p}$ is taken care of. If $G=\mathbf{Z} / \mathbf{p}^{\infty}$ for some prime $\mathbf{p}$, then $G * G=G$, so $\operatorname{dim}_{G}(A \cup B) \leq \operatorname{dim}_{G} A+\operatorname{dim}_{G} B+2$ by Theorem 1.3. By $\left[\mathrm{Dy}_{1}\right]$,

$$
\operatorname{dim}_{G}(A \cup B) \leq \operatorname{dim}_{G} A+\operatorname{dim}_{G} B+2
$$

for any torsion group $G$.

Consider a torsion-free abelian group $G$. Let $\mathcal{L}=\{\mathbf{p} \mid \mathbf{p} G \neq G\}$. By $\left[\mathrm{Dy}_{1}\right]$

$$
\operatorname{dim}_{\mathbf{Z}_{\mathcal{L}}} X \geq \operatorname{dim}_{G} X \geq \operatorname{dim}_{\mathbf{Z}_{\mathcal{L}}} X-1
$$

for any metrizable space $X$. Since $G \otimes \mathbf{Z}_{\mathcal{L}}=G$, applying Theorem 1.2 to $H=\mathbf{Z}_{\mathcal{L}}$ one gets

$$
\operatorname{dim}_{G}(A \cup B) \leq \operatorname{dim}_{G} A+\operatorname{dim}_{\mathbf{Z}_{\mathcal{L}}} B+1 \leq \operatorname{dim}_{G} A+\operatorname{dim}_{G} B+2 . \square
$$

Notice that the estimates of Theorem 1.4 cannot be improved. Indeed, Dranishnikov, Repovš and Ščepin [D-R-S] found an example such that $G=\mathbf{Q} / \mathbf{Z}$ and $\operatorname{dim}_{G}(A \cup B)=\operatorname{dim}_{G} A+\operatorname{dim}_{G} B+2$. The next result generalizes the method of $\left[\mathrm{Dy}_{3}\right]$ to produce such examples. 
Theorem 1.5. If $G \otimes G=0$, then for any $m \geq 2$ there is a subset $A$ of $S^{2 m+1}$ such that $\operatorname{dim}_{G} A \leq m-1$ and $\operatorname{dim}_{G}\left(S^{2 m+1}-A\right) \leq m$.

Proof. Notice that $G=\operatorname{Tor} G$ and $G$ is divisible. Let $H=\bigoplus\{\mathbf{Z} / \mathbf{p} \mid \mathbf{p}-$ Tor $G \neq 0\}$ and $G^{\prime}=\bigoplus\left\{\mathbf{Z} / \mathbf{p}^{\infty} \mid \mathbf{p}\right.$-Tor $\left.G \neq 0\right\}$. Then $G^{\prime} \otimes H=0$ and $\operatorname{dim}_{G} X=\operatorname{dim}_{G^{\prime}} X \leq$ $\operatorname{dim}_{H} X$ for any metrizable space $X$ (see [Dy 1 ). Thus, we may replace $G$ by $G^{\prime}$. We are going to use the following result of $\left[\mathrm{Dy}_{4}\right]$ :

Let $G$ be an abelian group and $m>0$. Let $\mathcal{G}$ be a countable family of countable abelian groups and let $D: \mathcal{G} \rightarrow \mathbf{Z}_{+}$be a function. The following conditions are equivalent:

1. For any $0 \neq a \in H_{m}(P ; G), P$ is a $C W$ complex, there is a compactum $X$ and a map $\pi: X \rightarrow P$ such that

$$
\begin{gathered}
\operatorname{dim} X=m, \\
\operatorname{dim}_{H} X \leq D(H) \text { for each } H \in \mathcal{G}, \\
a \in \operatorname{im}\left(\check{H}_{m}(X ; G) \rightarrow \check{H}_{m}(P ; G)\right) .
\end{gathered}
$$

2. $\widetilde{H}_{k}(K(H, D(H)) ; G)=0$ for all $k<m$ and all $H \in \mathcal{G}$.

In our application we have $\mathcal{G}=\{H\}$ and $D(H)=m-1$. Notice that $\widetilde{H}_{m-1}(K(H, m-1) ; G)=G \otimes H=0$. Choose a sequence $\left\{M_{i}\right\}_{i \geq 1}$ of open subsets of $S^{2 m+1}$ such that for any closed subset $C$ of $S^{2 m+1}$ and for any neighborhood $U$ of $C$ there is $i$ with $C \subset M_{i} \subset U$.

Given an element $a \in H_{m}\left(M_{i} ; G\right)$, there is a map $\pi: X \rightarrow P$ from a compactum $X$ such that $\operatorname{dim} X \leq m, \operatorname{dim}_{H} X \leq m-1, a \in \operatorname{im}\left(\check{H}_{m}(X ; G) \rightarrow \check{H}_{m}\left(M_{i} ; G\right)\right)$ and $\pi(X) \subset \operatorname{int} M_{i}$. Since $\pi$ is approximable by embeddings, we may simply assume $X \subset M_{i}$. Since each $H_{m}\left(M_{i}, G\right)$ is countable, one can find a sequence of compacta $X_{i, j} \subset M_{i}, j \geq 1, \operatorname{dim}_{H} X_{i, j} \leq m-1$, such that given an element $a \in H_{m}\left(M_{i} ; G\right)$ one can find its representative in $\check{H}_{m}\left(X_{i, j} ; G\right)$ for some $j$. Let $Y$ be the union of all $X_{i, j}$. Since $\operatorname{dim}_{H} Y \leq m-1$, there is a compactification $Y^{\prime}$ of $Y$ such that $\operatorname{dim}_{H} Y^{\prime} \leq m-1$ (apply Corollary 2.5 (a) or Shvedov's Theorem [Ku]). Let $A$ be a $G_{\delta}$ set in $S^{2 m+1}$ containing all the $X_{i, j}, i, j \geq 1$, such that $A$ is homeomorphic to a subset of $Y^{\prime}$, in particular $\operatorname{dim}_{H} A \leq m-1$. To show that $\operatorname{dim}_{G}\left(S^{2 m+1}-A\right) \leq m$ it suffices to prove $H^{m}\left(S^{2 m+1}-A ; G\right) \rightarrow H^{m}(C ; G)$ is an epimorphism for any compact subset $C$ of $S^{2 m+1}-A\left(S^{2 m+1}-A\right.$ is a countable union of compact sets). Given $\alpha \in H^{m}(C ; G)$, it can be represented by an element of $H_{m}\left(S^{2 m+1}-C ; G\right)$ (Alexander Duality - see [Sp], p.297). This element, in turn, belongs to the image of inclusion-induced $\check{H}_{m}\left(X_{i, j} ; G\right) \rightarrow H_{m}\left(S^{2 m+1}-C ; G\right)$ for some $(i, j)$. Dually, $\alpha$ extends over $S^{2 m+1}-X_{i, j} \supset S^{2 m+1}-A$. Thus, $\operatorname{dim}_{G}\left(S^{2 m+1}-A\right) \leq m$.

Remark. The main tool in the proof of Theorem 1.5 is a dual to the result of $\left[\mathrm{Dr}_{4}\right]$.

Conjecture 1.6. Suppose $A, B$ are subsets of a metrizable space. Then

$$
\operatorname{dim}_{G}(A \cup B) \leq \operatorname{dim}_{G} A+\operatorname{dim}_{G} B+1
$$

for any abelian torsion-free group $G$.

Remark. Any counterexample to the above Conjecture would produce a space $X=A \cup B$ and a torsion-free group $H$ (either $G$ or $G \otimes G$ ) such that $\operatorname{dim}_{H} X \neq$ $\sup \left\{\operatorname{dim}_{\mathbf{Z}_{\mathbf{p}}} X \mid \mathbf{p} H \neq H\right\}$, thus showing that Bockstein Theorem is not valid for metrizable spaces. 


\section{UNIVERSAL SPACES, COMPACTIFICATIONS AND COMPLETIONS}

This section is devoted to a partial solution to Problems 4-7. Up to now the only results have been obtained by A. Chigogidze [Ch] and I. A. Shvedov [Ku]:

Theorem (A. Chigogidze). Let $n \geq 0$, and let $G$ be an abelian group such that there exists a separable completely metrizable ANR-space homotopy equivalent to the Eilenberg-Mac Lane complex $K(G, n)$. Then, there exists a separable, completely metrizable space $X(G, n)$ such that the following conditions are equivalent for each metrizable compactum $K$ :

(i) $\operatorname{dim}_{G} K \leq n$,

(ii) $K$ embeds into $X(G, n)$.

Theorem (I. A. Shvedov). Let $X$ be a separable and metrizable space. If $K_{i} \in$ $A E(X), i \geq 1$, are finite polyhedra, then there exists a metrizable compactification $c(X)$ of $X$ such that $K_{i} \in A E(c(X))$ for all $i \geq 1$.

Our goal is to prove a result which simultaneously generalizes both Shvedov's Theorem and Chigogidze's Theorem. First, recall the notion of a universal space:

Definition. Given a class $\mathcal{C}$ of topological spaces, $X \in \mathcal{C}$ is called a universal space of $\mathcal{C}$ provided any $Y \in \mathcal{C}$ embeds in $X$.

Lemma 2.1. Suppose $u: K \rightarrow L$ is a map from a $C W$ complex $K$ to a compact metrizable space L. Given a compactum $X$ and a map $g: C \rightarrow K, C$ closed in $X$, there is a compactum $X^{\prime}$ and a map $\pi: X^{\prime} \rightarrow X$ with the following properties:

a. Given $f: Y \rightarrow X, K \in A E(Y)$, there is $f^{\prime}: Y \rightarrow X^{\prime}$ with $f=\pi f^{\prime}$,

b. There is $g^{\prime}: X^{\prime} \rightarrow L$ with $g^{\prime} \mid \pi^{-1}(C)=u g \pi$.

Proof. Extend $g$ to $G: D \rightarrow K$, where $D$ is a closed subset of $X$ containing $C$ in its interior. Let $X^{\prime}=(X-\operatorname{int}(D)) \times L \cup\{(x, u G(x)) \mid x \in D\} . \pi: X^{\prime} \rightarrow X$ is the projection onto $X$ and $g^{\prime}: X^{\prime} \rightarrow L$ is the projection onto $L$. Notice that $\pi^{-1}(C)=\{(x, u g(x)) \mid x \in C\}$. Hence, $g^{\prime}(x, u g(x))=u g(x)=u g \pi(x, u g(x))$. This verifies Condition (b).

To verify Condition (a), extend $G f \mid f^{-1}(D)$ to $G^{\prime}: Y \rightarrow K$ and define $f^{\prime}(y)=$ $\left(f(y), u G^{\prime}(y)\right)$ for $y \in Y$. To check that im $f^{\prime} \subset X^{\prime}$, notice that if $f(y) \in D$, then $u G\left(f(y)=u G^{\prime}(y)\right.$.

Lemma 2.2. Suppose $u: K \rightarrow L$ is a map from a countable $C W$ complex $K$ to a compact metrizable space $L$. Given a compactum $X$, there is a compactum $X^{\prime}$ and a map $\pi: X^{\prime} \rightarrow X$ with the following properties:

a. Given $f: Y \rightarrow X, K \in A E(Y)$, there is $f^{\prime}: Y \rightarrow X^{\prime}$ with $f=\pi f^{\prime}$,

b. For any $g: C \rightarrow K, C$ being closed in $X$, there is $g^{\prime}: X^{\prime} \rightarrow L$ with $g^{\prime}\left|\pi^{-1}(C) \approx u g \pi\right| \pi^{-1}(C)$.

Proof. Choose a sequence of closed subspaces $D_{i}, i \geq 1$, of $X$ with the property that for any closed set $C$ of $X$ and for any open set $U$ containing $C$ there is $i$ with $C \subset D_{i} \subset U$. A simple way of doing it is to take a countable basis $\mathcal{B}$ of $X$ and enumerate closures of unions of finite subfamilies of $\mathcal{B}$. Enumerate $\bigcup_{s=1}^{\infty}\left[D_{s}, K\right]$ as $\left[f_{j}: C_{j} \rightarrow K\right], j \geq 1$ (thus, each $C_{j}$ is one of the $D_{s}, s \geq 1$ ). Using Lemma 2.1, construct by induction an inverse sequence $\left\{X_{i}, \pi_{i}^{i+1}\right\}$ such that

1. $X_{1}=X$ 
2. given $f: Y \rightarrow X_{i}, K \in A E(Y)$, there is $f^{\prime}: Y \rightarrow X_{i+1}$ with $f=\pi_{i}^{i+1} f^{\prime}$,

3. there is an extension $g_{n}: X_{n+1} \rightarrow L$ of the composition $\left(\pi_{1}^{n+1}\right)^{-1}\left(C_{n}\right) \rightarrow$ $C_{n} \rightarrow K \rightarrow L$.

Let $X^{\prime}$ be the limit of $\left\{X_{i}, \pi_{i}^{i+1}\right\}$ and let $\pi: X^{\prime} \rightarrow X=X_{1}$ be the natural projection. It is clear that Condition (a) is satisfied. Suppose $g: C \rightarrow K, C$ being closed in $X$, and choose $n$ such that $C \subset C_{n}$ and $f_{n} \mid C \approx g$. Notice that the composition $g^{\prime}$ of the natural projection $X^{\prime} \rightarrow X_{n+1}$ and $g_{n}: X_{n+1} \rightarrow L$ satisfies $g^{\prime}\left|\pi^{-1}(C) \approx u g \pi\right| \pi^{-1}(C)$.

Theorem 2.3. Suppose $u: K \rightarrow L$ is a map from a countable $C W$ complex $K$ to a compact metrizable space $L$. Given a compactum $X$, there is a compactum $X^{\prime}$ and a map $\pi: X^{\prime} \rightarrow X$ with the following properties:

a. Given $f: Y \rightarrow X, K \in A E(Y)$, there is $f^{\prime}: Y \rightarrow X^{\prime}$ with $f=\pi f^{\prime}$,

b. Given $g: C \rightarrow K, C$ closed in $X^{\prime}$, there is $g^{\prime}: X^{\prime} \rightarrow L$ with $g^{\prime} \mid C$ homotopic to ug.

Proof. Using Lemma 2.2, construct by induction an inverse sequence $\left\{X_{i}, \pi_{i}^{i+1}\right\}$ such that

1. $X_{1}=X$

2. given $f: Y \rightarrow X_{i}, K \in A E(Y)$, there is $f^{\prime}: Y \rightarrow X_{i+1}$ with $f=\pi_{i}^{i+1} f^{\prime}$,

3. for any $g: C \rightarrow K, C$ being closed in $X_{i}$, there is $g^{\prime}: X_{i+1} \rightarrow L$ with $g^{\prime} \mid\left(\pi_{i}^{i+1}\right)^{-1}(C) \approx u g \pi_{i}^{i+1}$.

Let $X^{\prime}$ be the limit of $\left\{X_{i}, \pi_{i}^{i+1}\right\}$ and let $\pi: X^{\prime} \rightarrow X=X_{1}$ be the natural projection. It is clear that Condition (a) is satisfied. Suppose $g: C \rightarrow K, C$ being closed in $X^{\prime}$, and let $C_{n}=\pi_{n}(C)$ for each $n, \pi_{n}: X^{\prime} \rightarrow X_{n}$ being the natural projection. By [M-S, Theorem 9 on p.65] there is $n$ and $g_{n}: C_{n} \rightarrow K$ such that $g_{n} \pi_{n} \mid C \approx g$. Let $g_{n}^{\prime}: X_{n+1} \rightarrow L$ be such that $g_{n}^{\prime} \mid\left(\pi_{n}^{n+1}\right)^{-1}\left(C_{n}\right) \approx u g_{n} \pi_{n}^{n+1}$. Put $g^{\prime}=g_{n}^{\prime} \pi_{n+1}$.

In order to apply Theorem 2.3 we need the following fact:

Lemma 2.4. Given a sequence $\left\{K_{i}\right\}_{i \geq 1}$ of $C W$ complexes, let $K^{\prime}$ be the wedge of $\left\{K_{i}\right\}_{i \geq 1}$ and let $K$ be the subcomplex $(\{*\} \times R) \cup \bigcup_{i=1}^{\infty} K_{i} \times\{i\}$ of $K^{\prime} \times R$, where $*$ is the base point of $K^{\prime}$. If $X$ is a metrizable space, then $K \in A E(X)$ is equivalent to $K_{i} \in A E(X)$ for all $i$.

Proof. Since each $K_{i}$ is a retract of $K$, it suffices to show that $K_{i} \in A E(X)$ for all $i$ implies $K \in A E(X)$. Suppose $A$ is a closed subset of $X$ and $f: A \rightarrow K$. Extend the composition $A \rightarrow K \rightarrow R$ to $\alpha: X \rightarrow R$. If $\alpha(x)=i+1 / 2$, define $f(x)=(*, i+1 / 2)$. Since $K_{i} \times\{i\} \cup\{*\} \times[i-1 / 2, i+1 / 2] \in A E(X), f$ can be extended over $\alpha^{-1}[i-1 / 2, i+1 / 2]$.

Corollary 2.5. Suppose $\left\{K_{i}\right\}_{i \geq 1}$ is a sequence of $C W$ complexes homotopy dominated by finite $C W$ complexes. Then

a. Given a separable, metrizable space $Y$ such that $K_{i} \in A E(Y), i \geq 1$, there exists a metrizable compactification $c(Y)$ of $Y$ such that $K_{i} \in A E(c(Y))$, $i \geq 1$.

b. There is a universal space of the class

$$
\mathcal{C}=\left\{Y \text { is compact metrizable } \mid K_{i} \in A E(Y) \text { for all } i \geq 1\right\} \text {. }
$$


c. There is a completely metrizable and separable space $Z$ such that $K_{i} \in A E(Z)$ for all $i \geq 1$ with the property that any completely metrizable and separable space $Z^{\prime}$ with $K_{i} \in A E\left(Z^{\prime}\right)$ for all $i \geq 1$ embeds in $Z$ as a closed subset.

Proof. Let $X$ be the Hilbert cube. Without loss of generality we may assume that each $K_{i}$ is countable. Let $K$ be the wedge of $K_{i}, i \geq 1$, and $L$ be the compact wedge of $L_{i}\left(L_{i}\right.$ a finite complex homotopy dominating $\left.K_{i}\right), i \geq 1$. Choose maps $u_{i}: K_{i} \rightarrow L_{i}$ and $d_{i}: L_{i} \rightarrow K_{i}$ such that $d_{i} u_{i} \approx i d$. Define $u: K \rightarrow L$ to be the wedge of $u_{i}, i \geq 1$. Let $\pi: X^{\prime} \rightarrow X$ be the map as in Theorem 2.3. Notice that $X^{\prime}$ is a universal space for the class

$$
\mathcal{C}=\left\{Y \text { is compact metrizable } \mid K_{i} \in A E(Y) \text { for all } i \geq 1\right\} .
$$

Indeed, any separable and metrizable space embeds in $X$. Choose a lift $g: Y \rightarrow X^{\prime}$ of the inclusion $Y \hookrightarrow X$. In particular, $g(Y)$ is homeomorphic to $Y$. If $Y$ is not compact, we put $c(Y)=\operatorname{cl}(g(Y))$. Finally, $Z$ is defined as $\pi^{-1}(\sigma)$, where $\sigma$ is any subset of $X$ homeomorphic to the countable product of lines. To complete the proof we need to show that $K_{i} \in A E\left(X^{\prime}\right)$ for all $i$. Suppose $f: A \rightarrow K_{i}$ and $A$ is closed in $X^{\prime}$. There is $h: X^{\prime} \rightarrow L$ with $h \mid A \approx u f$. Notice that there is a retraction $r_{i}: L \rightarrow L_{i}$. Define $f^{\prime}=d_{i} r_{i} h: X^{\prime} \rightarrow K_{i}$ and notice that $f^{\prime} \mid A \approx f$.

Remark. Part (c) of Corollary 2.5 was proved by A. Chigogidze $\left[\mathrm{Ch}_{1}\right]$ and A. Waśko [Was] in the case when $K_{i}$ is the $n$-dimensional sphere ( $n$ is fixed).

Theorem 2.6. Suppose $K$ is a countable $C W$ complex, $X$ is a completely metrizable space and $c(X)$ is a metrizable compactification of $X$. There is a map $\pi$ : $\left(c\left(X^{\prime}\right), X^{\prime}\right) \rightarrow(c(X), X)$ with the following properties:

a. $X^{\prime}$ is completely metrizable and $c\left(X^{\prime}\right)$ is a metrizable compactification of $X^{\prime}$.

b. Given $f: Y \rightarrow X, K \in A E(Y)$, there is $f^{\prime}: Y \rightarrow X^{\prime}$ with $f=\pi f^{\prime}$,

c. Given $g: C \rightarrow K, C$ closed in $c\left(X^{\prime}\right)$, there is $g^{\prime}: X^{\prime} \rightarrow K$ with $g^{\prime} \mid C \cap X^{\prime}$ homotopic to $g$.

Proof. We may assume that $K$ is locally compact (replace $K$ by the telescope $\bigcup^{\infty} K_{k} \times[k-1, k]$, where $K_{i}, i \geq 1$ is an increasing sequence of finite subcomplexes of $K$ whose union is $K)$. Let $L$ be the one point compactification of $K$ and let $u: K \rightarrow L$ be the inclusion. We need the following variant of Lemma 2.1:

Claim 1. Suppose $Z$ is a completely metrizable space and $c(Z)$ is a metrizable compactification of $Z$. Suppose $g: C \rightarrow K$ is a map and $C$ is a closed subset of $c(Z)$. There is a map $\pi:\left(c\left(Z^{\prime}\right), Z^{\prime}\right) \rightarrow(c(Z), Z)$ with the following properties:

a. $c\left(Z^{\prime}\right)$ is a compactification of a completely metrizable space $Z^{\prime}$,

b. Given $f: Y \rightarrow Z, K \in A E(Y)$, there is $f^{\prime}: Y \rightarrow Z^{\prime}$ with $f=\pi f^{\prime}$,

c. There is $g^{\prime}:\left(c\left(Z^{\prime}\right), Z^{\prime}\right) \rightarrow(L, K)$ with $g^{\prime}\left|\pi^{-1}(C)=g \pi\right| \pi^{-1}(C)$.

Proof of Claim 1. Extend $g$ to $G: D \rightarrow K$, where $D$ is a closed subset of $c(X)$ containing $C$ in its interior. Let $T=(c(X)-\operatorname{int}(D)) \times L \cup\{(x, G(x)) \mid x \in D\}$, $Z^{\prime}=T \cap X \times K$ and $c\left(Z^{\prime}\right)=c l\left(Z^{\prime}\right) . \pi: c\left(Z^{\prime}\right) \rightarrow c(Z)$ is the projection onto $c(Z)$ and $g^{\prime}: c\left(Z^{\prime}\right) \rightarrow L$ is the projection onto $L$. Notice that $\pi^{-1}(C)=\{(x, g(x)) \mid x \in C\}$. Hence, $g^{\prime}(x, g(x))=g(x)=g \pi(x, g(x))$. This verifies Condition (c).

To verify Condition (b), extend $G f \mid f^{-1}(D)$ to $G^{\prime}: Y \rightarrow K$ and define $f^{\prime}(y)=$ $\left(f(y), G^{\prime}(y)\right)$ for $y \in Y$. To check that im $f^{\prime} \subset X^{\prime}$, notice that if $f(y) \in D$, then $G(f(y))=G^{\prime}(y)$. 
Also, we need the following variant of Lemma 2.2:

Claim 2. Suppose $Z$ is a completely metrizable space and $c(Z)$ is a metrizable compactification of $Z$. There is a map $\pi:\left(c\left(Z^{\prime}\right), Z^{\prime}\right) \rightarrow(c(Z), Z)$ with the following properties:

a. $c\left(Z^{\prime}\right)$ is a compactification of a completely metrizable space $Z^{\prime}$,

b. Given $f: Y \rightarrow Z, K \in A E(Y)$, there is $f^{\prime}: Y \rightarrow Z^{\prime}$ with $f=\pi f^{\prime}$,

c. Any $g: C \rightarrow K, C$ being closed in $c(Z)$, is homotopic to $h: C \rightarrow K$ such that there is an extension $g^{\prime}:\left(c\left(Z^{\prime}\right), Z^{\prime}\right) \rightarrow(L, K)$ of $h \pi \mid \pi^{-1}(C)$.

Proof of Claim 2. Choose a sequence of closed subspaces $D_{i}, i \geq 1$, of $c(Z)$ with the property that for any closed set $C$ of $c(Z)$ and for any open set $U$ containing $C$ there is $i$ with $C \subset D_{i} \subset U$. Enumerate $\bigcup_{s}\left[D_{s}, K\right]$ as $\left[f_{j}: C_{j} \rightarrow K\right], j \geq 1$. Using

Claim 1 , construct by induction an inverse sequence $\left\{\left(c\left(Z_{i}\right), Z_{i}\right), \pi_{i}^{i+1}\right\}$ such that

1. $\left(c\left(Z_{1}\right), Z_{1}\right)=(c(Z), Z)$,

2. given $f: Y \rightarrow Z_{i}, K \in A E(Y)$, there is $f^{\prime}: Y \rightarrow Z_{i+1}$ with $f=\pi_{i}^{i+1} f^{\prime}$,

3. there is an extension $g_{n}:\left(c\left(Z_{n+1}\right), Z_{n+1}\right) \rightarrow(L, K)$ of the composition $\left(\pi_{1}^{n+1}\right)^{-1}\left(C_{n}\right) \rightarrow C_{n} \rightarrow K \rightarrow L$.

Let $\left(c\left(Z^{\prime}\right), Z^{\prime}\right)$ be the limit of $\left\{\left(c\left(Z_{i}\right), Z_{i}\right), \pi_{i}^{i+1}\right\}$ and $\pi:\left(c\left(Z^{\prime}\right), Z^{\prime}\right) \rightarrow(c(Z), Z)=$ $\left(c\left(Z_{1}\right), Z_{1}\right)$ the natural projection. It is clear that Conditions (a-b) is satisfied. Suppose $g: C \rightarrow K, C$ being closed in $Z$, and choose $n$ such that $C \subset C_{n}$ and $f_{n} \mid C \approx g$. Notice that the composition $g^{\prime}$ of the natural projection $\left(c\left(Z^{\prime}\right), Z^{\prime}\right) \rightarrow$ $\left(c\left(Z_{n+1}\right), Z_{n+1}\right)$ and $g_{n}:\left(c\left(Z_{n+1}\right), Z_{n+1}\right) \rightarrow(L, K)$ is an extension $f_{n} \pi \mid \pi^{-1}(C)$.

Using Claim 2, construct by induction an inverse sequence $\left\{\left(c\left(X_{i}\right), X_{i}\right), \pi_{i}^{i+1}\right\}$ such that

1. $\left(c\left(X_{1}\right), X_{1}\right)=(c(X), X)$,

2. given $f: Y \rightarrow X_{i}, K \in A E(Y)$, there is $f^{\prime}: Y \rightarrow X_{i+1}$ with $f=\pi_{i}^{i+1} f^{\prime}$,

3. any $g: C \rightarrow K, C$ being closed in $c\left(X_{i}\right)$, is homotopic to $h: C \rightarrow K$ such that there is an extension $g^{\prime}:\left(c\left(X_{i+1}\right), X_{i+1}\right) \rightarrow(L, K)$ of $h \pi_{i}^{i+1} \mid\left(\pi_{i}^{i+1}\right)^{-1}(C)$

Let $\left(c\left(X^{\prime}\right), X^{\prime}\right)$ be the limit of $\left\{\left(c\left(X_{i}\right), X_{i}\right), \pi_{i}^{i+1}\right\}$ and let

$$
\pi:\left(c\left(X^{\prime}\right), X^{\prime}\right) \rightarrow(c(X), X)=\left(c\left(X_{1}\right), X_{1}\right)
$$

be the natural projection. It is clear that Conditions (a-b) are satisfied. Suppose $g: C \rightarrow K, C$ being closed in $c\left(X^{\prime}\right)$, and let $C_{n}=\pi_{n}(C)$ for each $n, \pi_{n}: c\left(X^{\prime}\right) \rightarrow$ $c\left(X_{n}\right)$ being the natural projection. By [M-S, Theorem 9 on p.65] there is $n$ and $g_{n}: C_{n} \rightarrow K$ such that $g_{n} \pi_{n} \mid C \approx g$. Let $g_{n}^{\prime}:\left(c\left(X_{n+1}\right), X_{n+1}\right) \rightarrow(L, K)$ be such that $g_{n}^{\prime} \mid\left(\pi_{n}^{n+1}\right)^{-1}\left(C_{n}\right) \approx g_{n} \pi_{n}^{n+1}$. Put $g^{\prime}=g_{n}^{\prime} \pi_{n+1}$.

Corollary 2.7. Suppose $\left\{G_{i}\right\}_{i \geq 1}$ is a sequence of countable abelian groups and $\left\{m_{i}\right\}_{i \geq 1}$ is a sequence of non-negative natural numbers. There exists a separable, completely metrizable space $Z$ and its metrizable compactification $c(Z)$ satisfying the following properties:

1. Any separable and metrizable space $C$ such that $\operatorname{dim}_{G_{i}} C \leq m_{i}$ for all $i$ embeds in $Z$ (as a closed subset if $C$ is completely metrizable),

2. Given a map $g: A \rightarrow K\left(G_{i}, m_{i}\right)$, $A$ being a closed subset of $c(Z), g \mid A \cap Z$ extends over $Z$.

The results of this section seem to suggest a connection between existence of compactifications preserving cohomological dimension and the existence of universal 
spaces. Since, generally speaking, compactifications do not exist (see [Dr 3$],\left[\mathrm{D}-\mathrm{W}_{2}\right]$, $\left[\mathrm{Dy}_{2}\right]$ and $\left.[\mathrm{Ka}]\right)$, one is justified in suggesting the following conjectures:

Conjecture 2.8. Suppose $K$ is a countable $C W$ complex. If the class of compacta

$$
\{X \mid K \in A E(X)\}
$$

has a universal space, then $K$ is homotopy dominated by a finite $C W$ complex.

Conjecture 2.9. If $K$ is a countable $C W$ complex, then there is a universal space in the class of separable metric spaces

$$
\{X \mid K \in A E(X)\}
$$

Conjecture 2.10. If $K$ is a countable $C W$ complex and $K \in A E(X), X$ being a separable metric space, then there is a completion $X^{\prime}$ of $X$ with $K \in A E\left(X^{\prime}\right)$.

\section{Dranishnikov's TheOREM FOR METRIZABlE SPACES}

The purpose of this section is to generalize a result of A.N.Dranishnikov [ $\left.\mathrm{Dr}_{2}\right]$ stating that if a CW complex $K$ is an absolute extensor of a compactum $X$, then $\operatorname{dim}_{H_{m}(K ; \mathbf{Z})} X \leq m$ for all $m>0$. We need the same result for metrizable spaces; at the same time our proof is conceptually simpler than that of $\left[\mathrm{Dr}_{2}\right]$.

Lemma 3.1. Suppose $f: X^{\prime} \rightarrow X$ is an open map of metrizable spaces such that card $f^{-1}(x) \leq k$ for all $x \in X$ and some $k<\infty$. If $K \in A E(X)$ is a $C W$ complex, then $K \in A E\left(X^{\prime}\right)$.

Proof. By induction on $k$. For $k=1$ it amounts to the fact that $K \in A E(X)$ and $X^{\prime}$ open in $X$ imply that $K \in A E\left(X^{\prime}\right)$ (see [Dr, , Proposition 2] or Proposition 1.1). Suppose Lemma 3.1 holds for $k=n$ and consider $k=n+1$. Let $A=\{x \in$ $X \mid$ card $\left.f^{-1}(x) \leq n\right\}$. Notice that $A$ is closed in $X^{\prime}$. Given a map $g: C \rightarrow K$, $C$ closed in $X^{\prime}$, one can extend it over a neighborhood $U$ of $A \cup C$. Choose a neighborhood $V$ of $A \cup C$ in $U$ with $\operatorname{cl}(V) \subset U$. Given $x \in X^{\prime}-A$, there is an open neighborhood $U_{x}$ of $x$ in $X^{\prime}$ such that $f \mid U_{x}: U_{x} \rightarrow f\left(U_{x}\right)$ is a homeomorphism. Choose a locally finite cover $\left\{F_{s}\right\}_{s \in S}$ of $X^{\prime}$ consisting of closed sets and refining $\{V\} \cup\left\{U_{x} \mid x \in X^{\prime}-A\right\}$. We may assume that the set $S^{\prime}=\left\{s \in S \mid F_{s} \not \subset V\right\}$ is well-ordered. Suppose $g^{\prime}: \operatorname{cl}(V) \cup \bigcup\left\{F_{s} \mid s<t\right\} \rightarrow K$ is an extension of $g$. Since $K \in A E\left(F_{t}\right)$, one can extend $g^{\prime}$ over $c l(V) \cup \bigcup\left\{F_{s} \mid s \leq t\right\}$. By transfinite induction there is an extension $g^{\prime}: X^{\prime} \rightarrow K$ of $g$.

Definition. Given a space $X$ and $k>0$, the $k$-th symmetric product $S P^{k}(X)$ of $X$ is the space of orbits of the action of the symmetric group $S_{k}$ on $X^{k}$. Points of $S P^{k}(X)$ will be written in the form $\sum_{i=1}^{k} x_{i} . S P^{k}(X)$ is equipped with the quotient topology given by the natural map $\pi: X^{k} \rightarrow S P^{k}(X)$. If $X$ is metrizable, then $\pi: X^{k} \rightarrow S P^{k}(X)$ is both open and closed (see p. 255 of [D-T]), so $S P^{k}(X)$ is metrizable, too.

If $X$ has a base point $a$, then $S P^{k}(X)$ has $\sum_{i=1}^{k} a$ as its base point; this base point will be denoted by $a$, too. Notice that there is a natural inclusion $i: S P^{n}(X) \rightarrow$ 
$S P^{k}(X)$ for all $n<k$. It is given by the formula $i\left(\sum_{i=1}^{n} x_{i}\right)=\sum_{i=1}^{n} x_{i}+(k-n) a$. In this way, points of the form $\sum_{i=1}^{n} x_{i}, n<k$, can be considered as belonging to $S P^{k}(X)$.

The direct limit of $S P^{2}(X) \rightarrow \ldots \rightarrow S P^{n}(X) \rightarrow \ldots$ is denoted by $S P^{\infty}(X)$.

Lemma 3.2. Suppose $\left(X, x_{0}\right),(M, a)$ are pointed metrizable spaces and $f:\left(X, x_{0}\right)$ $\rightarrow\left(S P^{k}(M), a\right)$ is a map, $k \geq 1$. Let $\left(X^{\prime}, x_{0}^{\prime}\right)$ be the pull-back of the diagram

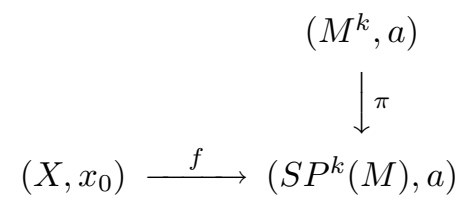

and let $\pi^{\prime}:\left(X^{\prime}, x_{0}^{\prime}\right) \rightarrow\left(X, x_{0}\right)$ be the natural projection. Then, $f^{*}:\left(X, x_{0}\right) \rightarrow$ $\left(S P^{k !}\left(X^{\prime}\right), x_{0}^{\prime}\right)$ defined by $f^{*}(x)=\sum_{y \in\left(\pi^{\prime}\right)^{-1}(x)} y$ is continuous.

Proof. Let $M^{k} \rightarrow S P^{k !}\left(M^{k}\right)$ be the map assigning to each element $m$ of $M^{k}$ the sum of all points obtained by permuting coordinates of $m$. It factors as $g \pi$ for some $g: S P^{k}(M) \rightarrow S P^{k !}\left(M^{k}\right)$. Let $i: X \times S P^{k !}\left(M^{k}\right) \rightarrow S P^{k !}\left(X \times M^{k}\right)$ be the map defined by $i\left(x, \sum_{i=1}^{k} m_{i}\right)=\sum_{i=1}^{k}\left(x, m_{i}\right)$. Notice that $f^{*}(x)=i(x, g f(x))$ for all $x \in X$.

Lemma 3.3. Suppose $X$ is metrizable and $K \in A E(X)$ is a pointed $C W$ complex. Given a closed subset $A$ of $X$ and $g: A \rightarrow S P^{k}(K)$, there is an extension $g^{\prime}: X \rightarrow$ $S P^{k \cdot k !}(K)$ of $g$.

Proof. Assume $K$ is a pointed simplicial complex. Add a discrete base point to $A$ and map it to the base point of $S P^{k}(K)$. Given a simplicial complex $L$, we use $L_{w}$ to denote $L$ with the weak $(\mathrm{CW})$ topology and $L_{m}$ to denote $L$ with the metric topology (both are homotopy equivalent - see [M-S, Theorem 10 on p.302]). Let $C(K)$ be the cone over $K$. Since $S P^{k}\left(C(K)_{w}\right)$ is contractible $\left(S P^{k}\right.$ is a functor from the homotopy category, see [D-T]), there is an extension $G: X \rightarrow S P^{k}\left(C(K)_{w}\right)$ of the composition $A \rightarrow S P^{k}\left(K_{w}\right) \rightarrow S P^{k}\left(C(K)_{w}\right)$. Let $f: X^{\prime} \rightarrow X$ be the pull-back of the projection $C(K)_{m}^{k} \rightarrow S P^{k}\left(C(K)_{m}\right)$ under $G$. Thus, $X^{\prime}=\left\{\left(x, x_{1}, \ldots, x_{k}\right) \in\right.$ $\left.X \times C(K)_{m}^{k} \mid G(x)=\sum_{i=1}^{k} x_{i}\right\}$ and $f\left(x, x_{1}, \ldots, x_{k}\right)=x . X^{\prime}$ contains, as a closed set, the space $A^{\prime}$ obtained as a pull-back of $K_{m}^{k} \rightarrow S P^{k}\left(K_{m}\right)$ under $g$. Extend the natural projection $A^{\prime} \rightarrow K_{m}^{k}$ over $X^{\prime}$ and compose it with $K_{m}^{k} \rightarrow S P^{k}\left(K_{m}\right)$. The resulting map induces $S P^{k !}\left(X^{\prime}\right) \rightarrow S P^{k !}\left(S P^{k}\left(K_{m}\right)\right) \rightarrow S P^{k \cdot k !}\left(K_{m}\right)\left(S P^{k}\right.$ is a functor), which, when composed with $f^{*}: X \rightarrow S P^{k !}\left(X^{\prime}\right)$, is an extension of $g: A \rightarrow S P^{k}\left(K_{m}\right)$. Since $K_{w}$ and $K_{m}$ are homotopy equivalent, we are done.

Remark. If $X$ is a compactum, Dranishnikov $\left[\mathrm{Dr}_{2}\right]$ proves a stronger result, namely that $K \in A E(X)$ implies $S P^{k}(K) \in A E(X)$ for all $k>0$.

Theorem 3.4. Suppose $X$ is metrizable and $K \in A E(X)$ is a $C W$ complex. Then,

$$
\operatorname{dim}_{H_{m}(K ; \mathbf{Z})} X \leq m
$$

for all $m>0$. 
Proof. It suffices to show that $S P^{\infty}(K) \in A E(X)$ as $S P^{\infty}(K)$ homotopy dominates $K\left(H_{m}(K ; \mathbf{Z}), m\right)$ for each $m>0$ (see $\left.[\mathrm{D}-\mathrm{T}]\right)$. Replace $S P^{\infty}(K)$ by the telescope

$$
\bigcup_{k=1}^{\infty} S P^{k}(K) \times[k-1, k]
$$

and use Lemma 3.3.

Added in proof. W. Olszewski $\left[\mathrm{Ol}_{1}\right]$ solved Problem 4 by answering Conjecture 2.10 positively.

J. Dydak and J. Mogilski [D-M] answered Conjecture 2.9 positively for $K=$ $K(\mathbf{Z}, n)$.

W. Olszewski $\left[\mathrm{Ol}_{2}\right]$ expanded section 2 of this paper and solved Problem 6 by answering Conjecture 2.9 positively.

A. Dranishnikov $\left[\mathrm{Dr}_{5}\right]$ solved Problem 8 positively.

\section{REFERENCES}

[Bo] B. F. Bockstein, Homological invariants of topological spaces, I, (English translation in Amer. Math. Soc. Transl. 11 (1959)), Trudy Moskov. Mat. Obshch. 5 (1956), 3-80. (Russian) MR 18:813

$\left[\mathrm{Ch}_{1}\right]$ A. Chigogidze, Uncountable powers of the real line and the natural series and $n$-soft maps, Soviet Math. Dokl. 90 (1984), 342-345. MR 86m:54049

$\left[\mathrm{Ch}_{2}\right] \quad$ A. Chigogidze, A note on cohomological dimension, preprint.

[Do] A. Dold, Lectures on algebraic topology, Springer-Verlag, Berlin, 1972. MR 54:3685

$\left[\mathrm{Dr}_{1}\right] \quad$ A. N. Dranishnikov, Homological dimension theory, Russian Maht. Surveys 43:4 (1988), 11-62. MR 90e:55003

[Dr, 2 A. N. Dranishnikov, Extension of maps into $C W$ complexes, Math. USSR Sbornik 74 (1993), 47-56. MR 93a:55002

[Dr 3 A. N. Dranishnikov, Cohomological dimension is not preserved by Stone-Čech compactification, Comptes Rendus Bulgarian Acad. of Sci. 41 (1988), 9-10. (Russian) MR 90e:55002

[Dr 4 A. N. Dranishnikov, Alternative construction of compacta with different dimensions, Proceedings of the Graduate Workshop in Mathematics and its Applications in Social Sciences, Ljubljana University 1991, Ljubljana (Slovenia), pp. 33-36.

[Dr 5 A. N. Dranishnikov, On the mapping intersection problem, preprint.

[D-R] A. Dranishnikov and D. Repovš, The Urysohn-Menger Sum Formula: An improvement of the Dydak-Walsh theorem to dimension one, J.Austral. Math. Soc., Ser.A (to appear).

[D-R-S] A. Dranishnikov, D. Repovš and E. Ščepin, On the failure of the Urysohn-Menger sum formula for cohomological dimension, Proc. Amer. Math. Soc. 120 (1994), 1267-1270. MR 94f:55001

[D-S] J. Dydak and J. Segal, Shape theory: An introduction, Lecture Notes in Math., vol. 688, Springer Verlag, 1978, pp. 1-150. MR 80h:54020

[D-T] A. Dold and R. Thom, Quasifaserungen und Unendliche Symmetrische Produkte, Annals of Math. 67 (1958), 239-281. (German) MR 21:3855

[D-M] J. Dydak and J. Mogilski, Universal cell-like maps, Proceedings of AMS 122 (1994), 943-948. MR 95a:55003

$\left[\mathrm{D}-\mathrm{W}_{1}\right]$ J. Dydak and J. J. Walsh, Aspects of cohomological dimension for principal ideal domains, preprint.

$\left[\mathrm{D}-\mathrm{W}_{2}\right]$ J. Dydak and J. J. Walsh, Spaces without cohomological dimension preserving compactifications, Proceedings of the Amer. Math. Soc. 113 (1991), 1155-1162. MR 92c:54039

[Dy 1 J. Dydak, Cohomological dimension and metrizable spaces, Transactions of the Amer. Math. Soc. 337 (1993), 219-234. MR 93g:55001

[Dy 2 J. Dydak, 219-234; Compactifications and cohomological dimension, Topology and its Appl. 50 (1993), 1-10. MR 94c:55002 
[Dy3] J. Dydak, Union theorem for cohomological dimension: A simple counterexample, Proceedings of AMS 121 (1994), 295-297. MR 94g:55001

[Dy4] J. Dydak, Realizing dimension functions via homology, Topology and its Appl. 64 (1995), $1-7$.

[Fu] L. Fuchs, Infinite abelian groups, Academic Press, New York and London, 1970. MR 41:333

[Hu] S. T. Hu, Theory of retracts, Wayne State University Press, Detroit, 1965. MR 31:6202

[Ka] A. I. Karinski, On cohomological dimension of the Stone-Čech compactification, Vestnik Mosk. Univ., Ser. 1 Mat. 1991, no. 4, 8-11. (Russian)

[Ku] W. I. Kuzminov, Homological dimension theory, Russian Math. Surveys 23 (1968), 1-45. MR 39:2158

[M-S] S. Mardes̀ić and J. Segal, Shape theory, North-Holland, Amsterdam, 1982. MR 84b:55020

$\left[\mathrm{Ol}_{1}\right]$ W. Olszewski, Completion theorem for cohomological dimensions, Proc. Amer. Math. Soc. 123 (1995), 2261-2264. CMP 95:10

$\left[\mathrm{Ol}_{2}\right]$ W. Olszewski, Universal separable metrizable spaces for given cohomological dimension, preprint.

[Ru] L. R. Rubin, Characterizing cohomological dimension: The cohomological dimension of $A \cup B$, Topology and its Appl. 40 (1991), 233-263. MR 92g:55002

[R-S] L. R. Rubin and P. J. Schapiro, Cell-like maps onto non-compact spaces of finite cohomological dimension, Topology and its Appl. 27 (1987), 221-244. MR 89b:55002

[Sp] E. Spanier, Algebraic topology, McGraw-Hill, New York, 1966. MR 35:1007

[Su] D. Sullivan, Geometric Topology, Part I: Localization, Periodicity, and Galois Symmetry, M.I.T. Press, 1970. MR 58:13006a

[Wa] J. J. Walsh, Dimension, cohomological dimension, and cell-like mappings, Lecture Notes in Math. 870, 1981, pp. 105-118. MR 83a:57021

[Was] A. Waśko, Spaces universal under closed embeddings for finite dimensional complete metric spaces, Bull. London. Math. Soc. 18 (1986), 293-298. MR 87e:54084

[We] J.West, Open problems in infinite dimensional topology, in Open Problems in Topology, North-Holland, 1990. MR 90e:57070

[Wh] George W.Whitehead, Elements of homotopy theory, Springer-Verlag, 1978. MR 80b: 55001

Department of Mathematics, University of Tennessee, Knoxville, Tennessee 37996

E-mail address: dydak@math.utk.edu 